\title{
KRAS GENE MUTATION ANALYSIS IN EXPERIMENTALLY INDUCED HAMSTER BUCCAL POUCH CARCINOMA
}

\author{
Emad Soliman Mohammed *, Mohamed Mahmoud Ahmed *** and Mohamed Gomaa Attia Zouair ${ }^{* * * *}$
}

\begin{abstract}
The aim of the present study was directed to screen the oncogenic Kras gene mutation in 7,12-dimethylbenz[a]anthracene (DMBA) induced hamster buccal pouch(s) (HBP(s)) carcinoma. Material and methods: Ten Syrian male hamsters, five weeks old, weighing 80-120g were divided into two group(s) (G(s)). GI (negative control): Right HBP mucosa of 5 animals were painted with liquid paraffin alone three times a week for 18 weeks. GII: (DMBA treated group): Right HBP of 5 animals were painted with $0.5 \%$ DMBA in paraffin oil 3 times a week for 18 weeks. After termination of the experiment, the HBPs were excised and bisected with one section fixed in $10 \%$ neutral buffered formalin, routinely processed and embedded in paraffin blocks in order to be sectioned and examined histologically. The other sections of the fresh tissues were taken and stored at $-80^{\circ} \mathrm{C}$ for DNA extraction followed by amplification of the targeted sequence using the polymerase chain reaction (PCR) technique. Then, the amplified targeted sequences were sequenced using the chain termination method (Sanger method) with a small concentration of dideoxynucleotides to detect the possible presence of Kras gene mutation. Results: Histological sections, using H\&E stain, showed moderately to poorly differentiated SCC. DNA sequencing results revealed that Kras gene mutation was absent in all cases. Conclusion: Oncogenic activation of Kras gene is not important in DMBA induced HBP carcinoma.
\end{abstract}

KEYWORDS: HBP carcinoma, Kras gene.

\section{INTRODUCTION}

Oral cancer encompasses all malignancies originating in the oral cavity and ranks sixth among the overall incidence for the 10 most common sites worldwide and third in the developing countries ${ }^{(1)}$. According to reports of the international agency for research on cancer (IARC) for oral cancer, the annual incidence is higher around the world, which is over 300.000 diagnosed cases, and the annual mortality is about 145,000 deaths, of which $77 \%$ were in the less developed regions ${ }^{(2)}$. It has been reported that oral squamous cell carcinoma (OSCC) represents over $90 \%$ of oral cancer ${ }^{(3)}$. The use of carcinogeninduced animal models to study the mechanisms of carcinogenesis is warranted, since chemical agents appeared to be the dominant etiologic factor in various areas of the head and neck including oral cavity. One of the best characterized animal models for OSCC is the hamster buccal pouch (HBP) system of oral carcinogenesis model that closely correlates with sequential common events involved in the development of premalignant and malignant human oral cancers ${ }^{(4)}$. Chemical carcinogens such as 7,12-dimethylbenz[a]anthracene (DMBA) are commonly employed to initiate and promote neoplastic transformation in experimental animals ${ }^{(5)}$.

Kras gene is a proto-oncogene located on 12p12.1 and encodes a 188-amino acid residue with a molecular weight of $21.6 \mathrm{kDa}$. Kras, a small intracellular GTPase, normally functions in signal

\footnotetext{
* Assistant lecturer, Oral and Dental Pathology Department, Faculty of Dental Medicine, (Boys-Cairo), Al-Azhar University, Egypt.

** Professor, Oral and Dental Pathology Department, Faculty of Dental Medicine,(Boys-Cairo), Al-Azhar University, Egypt.

*** Professor and Head, Oral and Dental Pathology Department, Faculty of Dental Medicine, (Boys-Cairo), Al-Azhar University, Egypt.
} 
transduction cascades initiated by the binding of epidermal growth factor, hepatocyte growth factor, and insulin-like growth factors to their receptors ${ }^{(6)}$. When activated wild-type Kras protein binds guanosine triphosphate (GTP), this results in a conformational change that allows the protein to bind and activate over 20 known downstream effectors which exert many different effects, including apoptosis suppression, promotion of cell growth, cell transformation, angiogenesis, migration, and differentiation ${ }^{(7)}$. Kras protein serves as a mediator between extracellular ligand binding and intracellular transduction of signals from epithelial growth factor receptor (EGFR) to the nucleus. Therefore, aberrant expression of Kras might result in sustained EGFR signaling despite inhibition ${ }^{(8)}$. Somatic point mutations in Kras gene occur in multiple human malignancies, including approximately $90 \%$ of pancreatic, $30 \%$ of lung, $60 \%$ of thyroid, and $43 \%$ of colorectal carcinomas $^{(9)}$. A mutation in codon 12 or 13 in Kras gene leads to constitutive activation of the protein, regardless of upstream activating signals and consequently, unregulated proliferation and impaired differentiation ${ }^{(10)}$. In head and neck SCC (HNSCC), there had been only limited investigation of RAS family mutations with mutation frequencies varied between $0-50 \%$ and appeared to be dependent on the specific RAS gene and interestingly, geographic location of the study population ${ }^{(11-14)}$.

DNA sequencing is considered the gold standard for detection of mutations. The Sanger chaintermination method is commonly used in molecular diagnostic laboratories for Kras mutation testing (15). In this method, the incorporation of chemically modified nucleotides known as dideoxynucleotides terminates extension of the DNA strand at the point of incorporation. This results in a mixture of DNA fragments of various lengths. Each dideoxynucleotide (A, T, C, or G) is labeled with a different fluorescent dye, allowing their individual detection. The newly synthesized and labeled DNA fragments are separated by size through capillary gel electrophoresis. The fluorescence is detected by an automated sequence analyzer, and the order of nucleotides in the target DNA is depicted as a sequence electropherogram. Automated sequence analysis is relatively inexpensive and easy to perform and detects all mutations present in the gene sequence ${ }^{(16)}$. Hence, the main target of the present study was directed to screen the Kras gene mutation in DMBA induced HBP carcinoma.

\section{MATERIAL AND METHODS}

The Experimental animals used in the current study were golden Syrian hamsters. They were used as model for OSCC induction utilizing 7,12 DMBA as chemical carcinogen. After tissue preparation, various investigations: hematoxylin and eosin (H\&E) stain, and DNA sequencing using Sanger chain termination method for Kras mutation testing, were done.

\section{Material used:}

Animals: Ten Syrian male hamsters, five weeks old, weighing 80-120g were obtained from the animal house, Cairo University (Cairo, Egypt). The experimental animals were housed in standard cages with sawdust bedding under controlled environmental conditions of humidity $(30-40 \%)$, temperature $\left(20 \pm 2^{\circ} \mathrm{C}\right)$, and light (12-h light/12-h dark). All experimental animals were supplied with standard diet and water ad libitum. 7,12 DMBA (0.5\%) was obtained from Sigma-Aldrich company, dissolved in paraffin oil and used thrice a week for 18 weeks via painting using a number 4 camel's hair brush ${ }^{(5)}$.

Experimental design: Ten Syrian male hamsters, were divided into two Gs. GI (negative control): Right HBP mucosa of 5 animals were painted with liquid paraffin alone three times a week for 18 weeks. GII: (DMBA treated group): Right HBP mucosa of 5 animals were painted with $0.5 \%$ DMBA in paraffin oil using No. 4 camel hair brush 3 times a week for 18 weeks. 
Investigations: After termination of the experiment, the animals were sacrificed by cervical dislocation, HBPs were everted, excised and bisected with one section fixed in $10 \%$ neutral buffered formalin, routinely processed and embedded in paraffin blocks for preparation in order to be stained and examined microscopically. The other sections of the fresh tissues were taken and stored at $-80^{\circ} \mathrm{C}$ for DNA extraction, polymerase chain reaction (PCR) amplification, and DNA sequencing procedures.

For histopathological examination: The fixed specimens were dehydrated in an ascending ethanol series, embedded in paraffin wax to form paraffin blocks. Tissue sections of $4 \mu \mathrm{m}$ thickness on rotary microtome were cut, mounted on glass slides, processed, and stained with H\&E for light microscopic examination.

DNA was extracted from the fresh tissue sections, using a specialized kit, followed by amplification of the targeted sequence using the PCR technique. Then, the amplified targeted sequences were sequenced using the chain termination method (Sanger method) with a small concentration of dideoxynucleotides to detect the possible presence of gene mutation.

Protocol of DNA extraction: Extraction of DNA from animal tissues was done using the spin-column protocol of Qiagen (Biotechnology Company, Germany). The kit used was dneasy blood \& tissue kit. Briefly: $25 \mathrm{mg}$ of each tissue sample was cut into small pieces to enable more efficient lysis, 20 $\mu 1$ proteinase $\mathrm{K}$ was added. Then mixed thoroughly by vortexing, and incubated at $56^{\circ} \mathrm{C}$ for 2 hours until the tissue is completely dissolved. $4 \mu 1 \mathrm{RNase}$ A $(100 \mathrm{mg} / \mathrm{ml})$ was added, mixed by vortexing, and incubated for 2 min at room temperature. Pipet the mixture from into the dneasy mini spin column placed in a $2 \mathrm{ml}$ collection tube. Centrifuged at $8000 \mathrm{rpm}$ for $1 \mathrm{~min}$. Flow-through and collection tube were discarded. Dneasy mini spin column was placed in a new $2 \mathrm{ml}$ collection tube, $500 \mu 1$ buffer AW was added, and centrifuged for 3 min at 14,000 rpm to dry the dneasy membrane. Flow-through and collection tube were discarded. Dneasy mini spin column was placed in a clean $2 \mathrm{ml}$ microcentrifuge tube, and pipet $200 \mu \mathrm{l}$ buffer AE directly onto the dneasy membrane. Incubated at room temperature for $1 \mathrm{~min}$, and then centrifuged for $1 \mathrm{~min}$ at $8000 \mathrm{rpm}$ to elute. Concentration and purity of the extracted DNA were measured using nanodrop2000 spectrophotometer. Measuring the concentration of DNA in the elute determined by its absorbance at $260 \mathrm{~nm}$. The ratio of the readings at $260 \mathrm{~nm}$ and 280 $\mathrm{nm}$ (A260/A280) provides an estimate of the purity of DNA with respect to contaminants that absorb UV, such as protein. pure DNA has an A260/A280 ratio of 1.8-2.0. PCR amplification of extracted DNA from animal tissues was done using the PCR master mix (2x) for 200 rxns, Thermo Fisher Scientific Inc. Description: PCR master mix is a $2 x$ concentrated solution of Taq DNA polymerase, dNTPs and all other components required for PCR, except DNA template and primers. This pre-mixed formulation saves time and reduces contamination due to a reduced number of pipetting steps required for PCR set up. The mix is optimized for efficient and reproducible PCR. Composition of the PCR master mix (2x): $0.05 \mathrm{U} / \mu \mathrm{L}$ Taq DNA polymerase, reaction buffer, $4 \mathrm{mM} \mathrm{MgCl} 2,0.4 \mathrm{mM}$ of each dNTP (dATP, dCTP, dGTP and dTTP). The primer sequences and conditions for DNA amplification with biometra thermal cycler are shown in table (1).

TABLE (1) The primer sequences and conditions for DNA amplification:

\begin{tabular}{|c|c|c|}
\hline Forward primer & 5'-GGCCTGCTGAAAATGACTGA & nealin \\
\hline Reverse primer & 5'-CTCTATCGTAGGATCATATT & $72^{\circ} \mathrm{C}$ for $60 \mathrm{~s}$ \\
\hline
\end{tabular}


To visualize the PCR products and to insure that the band of DNA amplified is the predicted size. The amplified samples were ran on $2 \%$ agarose gels using gel electrophoresis protocol. After the run, the gel with tray were removed and visualize PCR fragment bands using the E-Gel Imager. The gel photo was captured and saved.

PCR purification: This protocol is designed to purify double-stranded DNA fragments from PCR primers, nucleotides, polymerases, and salts using qiaquick spin columns protocol . The kit used was qiaquick PCR purification kit of Qiagen. Procedures: $500 \mu 1$ of buffer PB to $100 \mu 1$ PCR sample was added, and mixed until the mixture turned yellow. Qiaquick spin column was placed in a provided 2 $\mathrm{ml}$ collection tube. To bind DNA, the sample added to the qiaquick column and centrifuged at 13,000 rpm for 30-60 s. The flow-through discarded, and the qiaquick column was placed back into the same tube. To wash, $750 \mu 1$ Buffer PE was added to the qiaquick column and centrifuged at 13,000 rpm for 30-60 s. The flow-through discarded, and the qiaquick column was placed back in the same tube. The column was centrifuged for an additional $1 \mathrm{~min}$. Then, each qiaquick column was placed in a clean $1.5 \mathrm{ml}$ microcentrifuge tube. To elute DNA, $30 \mu 1$ of water was added to the center of the qiaquick membrane, and the column was centrifuged at 13,000 rpm for $1 \mathrm{~min}$.

DNA cycle sequencing: Cycle sequencing reaction was done using bigDye terminator protocol. The kit used was bigDye terminator v3.1 cycle sequencing kit. Procedures: The volume of cycle sequencing reaction was set (Table: 2 ). The tubes were placed in Genamp 9700 thermal cycler according to thermal profile: Denaturation $96^{\circ} \mathrm{C}$ for $1 \mathrm{~min}$. Annealing $50^{\circ} \mathrm{C}$ for $5 \mathrm{sec}$. Extension $60^{\circ} \mathrm{C}$ for $4 \mathrm{~min}$.
TABLE (2) Components of cycle sequencing reaction:

\begin{tabular}{|l|l|}
\hline Components & Volume \\
\hline BigDye terminator & $1 \mu \mathrm{l}$ \\
\hline 5x sequencing buffer & $1 \mu \mathrm{l}$ \\
\hline Primer & $1 \mu 1\left(5^{\prime}\right.$-GGCCTGCTGAAAATGACTGA) \\
\hline Template & $20 \mathrm{ng}$ \\
\hline Nuclease free water & Up to $10 \mu 1$ \\
\hline Total & $10 \mu 1$ \\
\hline
\end{tabular}

Capillary electrophoresis for purified cycle sequencing reaction: During capillary electrophoresis, the extension products of the cycle sequencing reaction enter the capillary of the 3500 genetic analyzer as a result of electrokinetic injection. A high voltage charge applied to the buffered sequencing reaction forces the negatively charged fragments into the capillaries. The extension products are separated by size based on their total charge. Procedures: $10 \mu 1$ of purified cycle sequenced DNA of each case was pipetted into the 96 well plate. Verified that each sample was positioned correctly in the bottom of each well. The plate was stored on ice until complete plate assembly, then the plate was loaded in the plate base and covered by the plate retainer of the 3500 genetic analyzer. The plate was placed in the autosampler of the analyzer and the door closed. Using the 3500 genetic analyzer software program, click link plate on the run screen to start running of the samples. Using applied biosystems data collection software, the fluorescence signals of all four colors of the bases were converted to digital data, then the data was recorded in a file. The sequencing files were analyzed automatically and immediately after the electrophoresis, analyzed sample data for each case was displayed as an electropherogram, a sequence of peaks in four colors. After data analysis of the capillary electrophoresis results, the nucleotide sequences of the chromatogram for normal control group were compared with the chromatogram nucleotide sequence results of the cancer bearing tissues of the other groups using the basic local 
alignment search tool (BLAST) of the national center for biotechnology information (NCBI). BLAST finds regions of similarity between biological sequences. The program compares nucleotide or protein sequences to sequence databases and calculates the statistical significance.

\section{RESULTS}

The gross observation results of HBP mucosa of GI was pink in color with smooth surface with no observable abnormalities (Fig.1:A). In GII, HBP mucosa showed various changes including variable sized nodules and exophytic papillary tumor masses with $100 \%$ tumor formation. Furthermore, eroded and ulcerative areas were seen throughout the tumor growth, with spontaneous bleeding (Fig.1:B).

Histopathological results: The tissue sections of HBP mucosa of experimental Gs showed variable results in regard to the histopathological results. In GI, histological sections, using $\mathrm{H} \& \mathrm{E}$ stain, revealed normal HBP mucosa, composed of thin stratified squamous epithelium without rete ridges with submucosal dense fibrous connective tissue and layer of longitudinal striated muscle fibers (Fig.2:A). In GII, histological sections, using H\&E stain, showed moderately to poorly differentiated SCC with deeply invading islands of tumor cells into the underlying connective tissue. The surface epithelium showed severe epithelial dysplasia (Fig.2:B)

DNA sequencing analysis: Using the printed photograph of the gel documentation system of agarose gel electrophoresis, the size of amplified DNA products was estimated. The product size was 123 bp in all cases (Fig.3).

\section{Capillary electrophoresis results: Kras} mutational analysis was done in 15 animals, including 5 animals from GI, 5 animals from GII, 5 animals from GIII. Wild-type Kras gene was found in all animals, with no mutation in sequences of codon 12 and codon 13 as shown in the chromatogram (Fig.4).

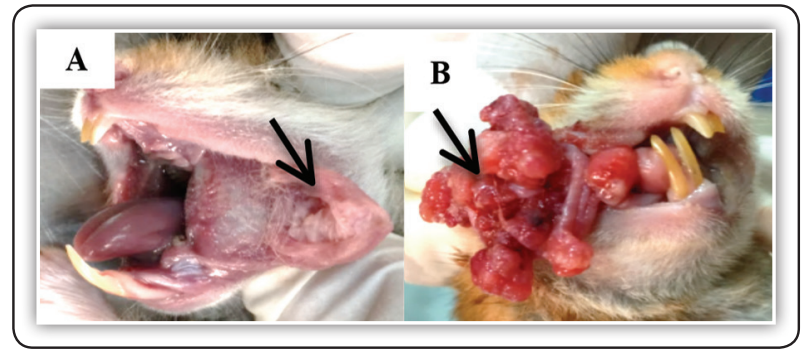

Fig.1(A): HBP mucosa of GI was pink in color with no observable abnormalities. Fig.1(B): HBP mucosa of GII showed variable sized nodular elevations and exophytic papillary tumor masses.

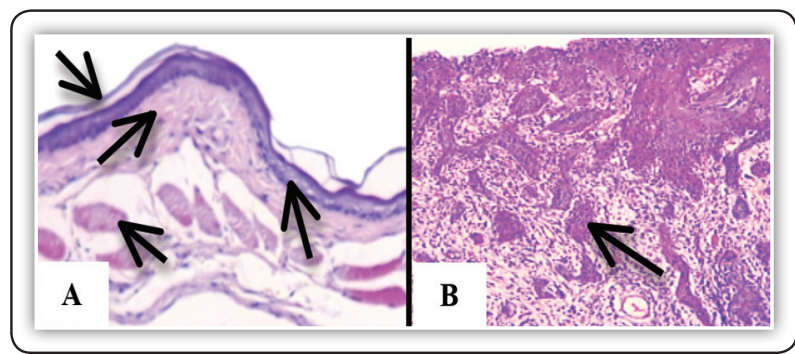

Fig.2(A): Photomicrograph of GI showed normal HBP mucosa, composed of thin stratified squamous epithelium without rete ridges with submucosal dense fibrous connective tissue and layer of longitudinal striated muscle fibers (arrows). Fig.2(C): Photomicrograph of GII showed moderately differentiated SCC with deeply invading islands of tumor cells into the underlying connective tissue (arrow).

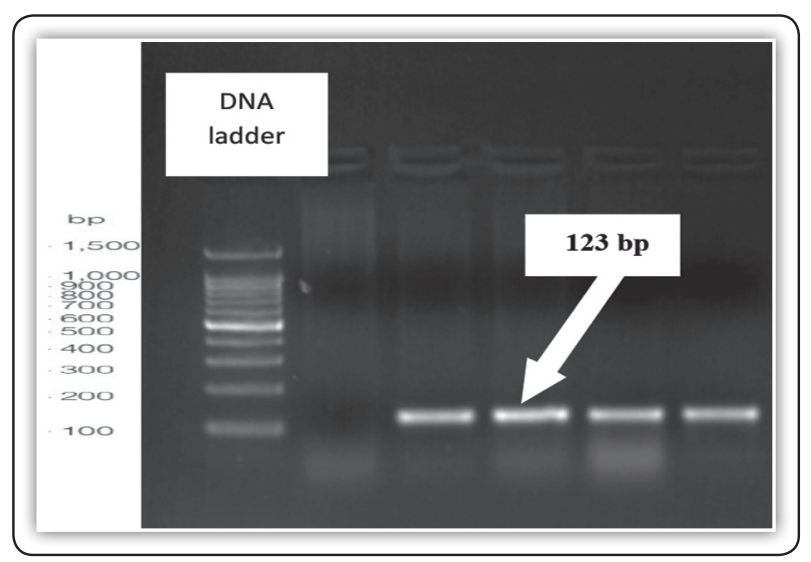

Fig. (3): Printed photograph of gel documentation system revealed agarose gel electrophoresis of PCR amplification products showing DNA ladder with known base pair sizes. Other lanes showing DNA of kras gene in 4 samples has product size of $123 \mathrm{bp}$. 


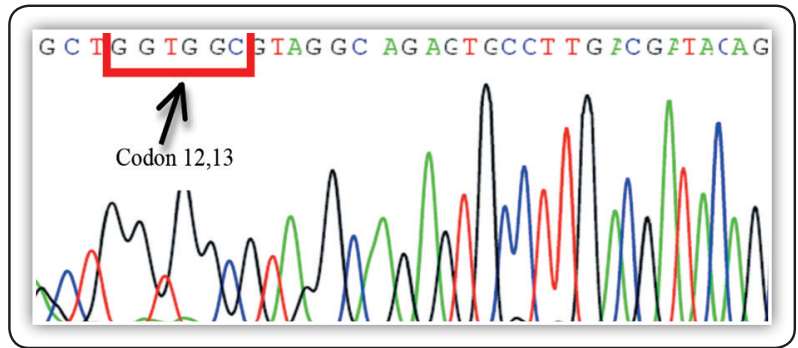

Fig. (4): Sequence chromatogram of HBP carcinoma displaying the wild-type Kras gene.

The nucleotide sequence of normal control group was:

TTGTGGGAGCTGGTGGCG-

TAGGGCAAGAGTGCCTTGACGATACAGCTAATTCAGAATCACTTTGTGGAT-

\section{GAATATGATCCTACGATAGAG}

The nucleotide sequence of cancer bearing animal from DMBA control group was:

TGGGAGCTGGTGGCG-

TAGGGCAAGAGTGCCTTGACGATACAGCTAATTCAGAATCACTTTGTGGATGAATATGATCCTACGATAGAGA

Using BLAST alignment (https/blast.ncbi.nlm. nih.gov/blast.cgi), the results of the cancer bearing cases were $100 \%$ identical with the control group (Fig.5).

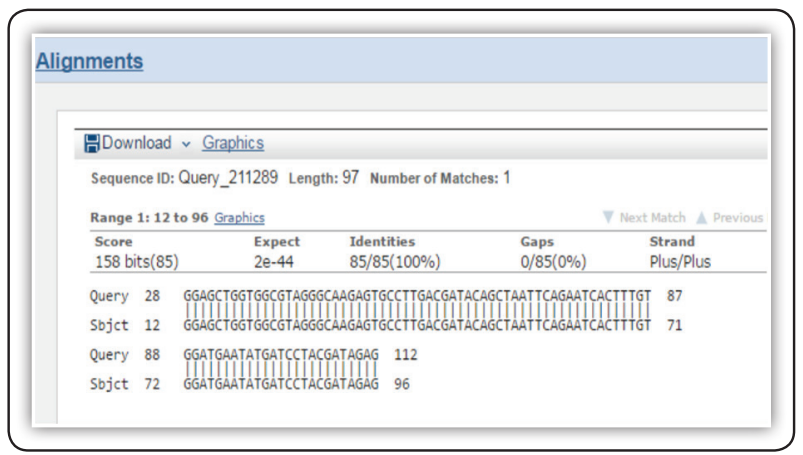

Fig. (5): BLAST results of two sequences showing no gaps with $100 \%$ identities.

\section{DISCUSSION}

The 5-year survival rate for OSCC is a mere 50\%, a figure that has remained relatively unchanged for decades ${ }^{(4)}$. Consequently, there has been an increasing focus on identifying key genetic players that may contribute to OSCC pathogenesis, with the overall goal of preventing onset and progression of disease. To date, numerous oncogenes and tumor suppressor genes have been implicated in the development of OSCC. The use of HBP system of oral carcinogenesis model is beneficial for deeper understanding of cancer biology, prevention and treatment. In the present study, the gross observation findings in GI showed no observable abnormalities of the hamster cheek pouch. After sacrificing, the buccal pouches length was about $5 \mathrm{~cm}$ for all hamsters with normal histological structures. Other studies reported the same findings ${ }^{(17,18)}$.

In the present study, the gross observation of GII revealed variable sized nodules and exophytic papillary tumor masses with $100 \%$ tumor formation. Furthermore, eroded and ulcerative areas were seen throughout the tumor growth, with spontaneous bleeding. These results are almost the same with that shown by other investigators ${ }^{(19-22)}$. Histopathological results of GII showed moderately to poorly differentiated SCC with deeply invading islands of tumor cells into the underlying connective tissue. The surface epithelium showed hyperkeratinization, and elongated drop-shaped rete ridges with areas of variable degrees of dysplasia. These results are in agreement with those of other investigators ${ }^{(22,23)}$. These observations might be attributed to DMBA induced overproduction of reactive oxygen species, chronic inflammation, oxidative modification of DNA bases, impairment in antioxidant defense system, defect in the activities of detoxification cascade and deregulated expression pattern of molecular markers are implicated in the promotion and progression of oral carcinogenesis ${ }^{(18)}$. 
In the present study, the DNA sequencing analysis for mutation detection in Kras gene using the chain termination method (Sanger method), the results revealed that wild-type Kras gene was found in all animals, with no mutation in sequences of codon 12 and codon 13. These results are in line with other studies which reported that, in HNSCC, the Kras mutations are infrequent and ranging from 0 to $6 \%{ }^{(13,14,24-26)}$. These results suggesting that oncogenic activation of Kras is not important in OSCC tumorigenesis or may be associated with other mechanisms other than small mutations, such as amplification and overexpression ${ }^{(11,27)}$. In conclusion, oncogenic activation of Kras gene is not important in DMBA induced HBP carcinoma.

\section{REFERENCES}

1. Jain M, Shah R, Chandolia B, Mathur A, Chauhan Y, Chawda J, et al. The Oral Carriage of Candida in Oral Cancer Patients of Indian Origin Undergoing Radiotherapy and/or Chemotherapy. J Clin Diagn Res 2016;10(2):17-20.

2. Ferlay J, Soerjomataram I, Dikshit R, Eser S, Mathers C, Rebelo $\mathrm{M}$, et al. Cancer incidence and mortality worldwide: sources, methods and major patterns in GLOBOCAN 2012. Int J Cancer 2015;136(5):359-86.

3. Krishna A, Singh S, Kumar V, Pal U. Molecular concept in human oral cancer. Natl J Maxillofac Surg 2015;6(1):9-15.

4. Vairaktaris E, Spyridonidou S, Papakosta V, Vylliotis A, Lazaris A, Perrea D, et al. The hamster model of sequential oral oncogenesis. Oral Oncol 2008;44(4):315-24.

5. Silvan S, Manoharan S, Baskaran N, Anusuya C, Karthikeyan S, Prabhakar M. Chemopreventive potential of apigenin in 7, 12-dimethylbenz (a) anthracene induced experimental oral carcinogenesis. Eur J Pharmacol 2011;670(2):571-77.

6. Malumbres M, Barbacid M. RAS oncogenes: the first 30 years. Nat Rev Cancer 2003;3(6):459-65.

7. Shackelford R, Whitling N, McNab P, Japa S, Coppola D. KRAS testing a tool for the implementation of personalized medicine. Genes Cancer 2012;3(7):459-66.

8. Boeckx C, Baay M, Wouters A, Specenier P, Vermorken J, Peeters M, et al. Anti-epidermal growth factor receptor therapy in head and neck squamous cell carcinoma: focus on potential molecular mechanisms of drug resistance. Oncologist 2013;18(7):850-64.
9. Boeckx C, Weyn C, Bempt I, Deschoolmeester V, Wouters A, Specenier P, et al. Mutation analysis of genes in the EGFR pathway in head and neck cancer patients: implications for anti-EGFR treatment response. BMC Res Notes 2014;7(1):337-45.

10. Shackelford R, Whitling N, McNab P, Japa S, Coppola D. KRAS testing a tool for the implementation of personalized medicine. Genes Cancer 2012;3(7):459-66.

11. Bruckman K, Schonleben F, Qiu W, Woo V, Su G. Mutational analyses of the BRAF, KRAS, and PIK3CA genes in oral squamous cell carcinoma. Oral Surg Oral Med Oral Pathol Oral Radiol Endod 2010; 110(5):632-37.

12. Athanasios C, Tsiatis A, Roy G, Michael J, Christopher D, James R, et al. Comparison of Sanger sequencing, pyrosequencing, and melting curve analysis for the detection of KRAS mutations. J Mol Diagn 2010;12(4):425-32.

13. Fujii S, Uryu H, Akashi K, Suzuki K, Yamazaki M, Tahara $\mathrm{M}$, et al. Clinical significance of KRAS gene mutation and epidermal growth factor receptor expression in Japanese patients with squamous cell carcinoma of the larynx, oropharynx and hypopharynx. Int $\mathrm{J}$ Clin Oncol 2013;18(3):454-63

14. Wang W, Chien Y, Wong Y, Lin Y, Lin J. Effects of KRAS mutation and polymorphism on the risk and prognosis of oral squamous cell carcinoma. Head Neck 2012;34(5):663-66

15. Monzon F, Ogino S, Hammond M, Halling K, Bloom K, Nikiforova M. The role of KRAS mutation testing in the management of patients with metastatic colorectal cancer. Arch Pathol Lab Med 2009;133(10):1600-06.

16. Ogino S, Kawasaki T, Brahmandam M, Yan L, Cantor M, Namgyal C, et al. Sensitive sequencing method for KRAS mutation detection by Pyrosequencing. J Mol Diagn 2005;7(3):413-21.

17. Casto B, Knobloch T, Galioto R, Yu Z, Accurso B, Warner B. Chemoprevention of oral cancer by lyophilized strawberries. Anticancer Res 2013;33(11):4757-66.

18. Balakrishnan S, Manoharan S, Alias L, Nirmal M. Effect of curcumin and ferulic acid on modulation of expression pattern of p53 and bcl-2 proteins in 7, 12-dimethylbenz [a] anthracene-induced hamster buccal pouch carcinogenesis. Indian J Biochem Biophys 2010; 47(1):7-12 .

19. Afifi M, El Sheikh S, Abdelsalam M, Ramadan H, Omar T, El Tantawi M, et al. Therapeutic efficacy of plasmonic photothermal nanoparticles in hamster buccal pouch carcinoma. Oral Surg Oral Med Oral Pathol Oral Radiol 2013;115(6):743-51 
20. Sophia J. Nimbolide, a neem limonoid inhibits Phosphatidyl Inositol-3 Kinase to activate Glycogen Synthase Kinase-3 $\beta$ in a hamster model of oral oncogenesis Sci Rep. 2016;6:22192-205.

21. Manoharan S, Rajasekaran D, Prabhakar M, Karthikeyan S, Manimaran A. Modulating effect of Enicostemma littorale on the expression pattern of apoptotic, cell proliferative, inflammatory and angiogenic markers during 7 , 12-Dimethylbenz (a) anthracene induced hamster buccal pouch carcinogenesis Toxicol Int. 2015;22(1):130-40.

22. Manoharan S, Wani S, Vasudevan K, Manimaran A, Prabhakar M, Karthikeyan S, et al. Saffron reduction of 7, 12-dimethylbenz [a] anthracene-induced hamster buccal pouch carcinogenesis. Asian Pac J Cancer Prev 2013;14(2):951-57.

23. Mohan K, Devaraj H, Prathiba D, Hara Y, Nagini S. Antiproliferative and apoptosis inducing effect of lactoferrin and black tea polyphenol combination on hamster buccal pouch carcinogenesis. Biochim Biophys Acta 2006; 1760(10):1536-44.

24. Cohen Y, Goldenberg N, Shalmon B, Shani T, Oren S, Amariglio N, et al. Mutational analysis of PTEN/PIK3CA/ AKT pathway in oral squamous cell carcinoma. Oral Oncol 2011;47(10):946-50.

25. Lopez F, Llorente J, Oviedo C, Vivanco B, Marcos C, Inclan $\mathrm{C}$, et al. Gene amplification and protein overexpression of EGFR and ERBB2 in sinonasal squamous cell carcinoma. Cancer 2012;118(7):1818-26.

26. Van Damme N, Deron P, Van Roy N, Demetter P, Bols A, Van Dorpe J, et al. Epidermal growth factor receptor and K-RAS status in two cohorts of squamous cell carcinomas. BMC cancer 2010;10:189-98.

27. Howell R, Wong F, Fenwick R. A transforming Kirsten ras oncogene in an oral squamous carcinoma. J Oral Pathol Med 1990; 19(7):301-05. 